Tohoku Math. J

56 (2004), 163-177

\title{
COHOMOGENEITY ONE ACTIONS ON NONCOMPACT SYMMETRIC SPACES WITH A TOTALLY GEODESIC SINGULAR ORBIT
}

\author{
JÜRGEN BERNDT AND HIROSHI TAMARU
}

(Received July 19, 2002, revised April 4, 2003)

\begin{abstract}
We classify all totally geodesic submanifolds of connected irreducible Riemannian symmetric spaces of noncompact type which arise as a singular orbit of a cohomogeneity one action on the symmetric space.
\end{abstract}

1. Introduction. The motivation for this paper is the classification problem for all isometric cohomogeneity one actions on connected irreducible Riemannian symmetric spaces of noncompact type. An isometric action of a connected Lie group on a Riemannian manifold is of cohomogeneity one if the codimension of a generic orbit is one. Two isometric cohomogeneity one actions on a Riemannian manifold are orbit equivalent if there exists an isometry of the manifold mapping the orbits of one of these actions onto the orbits of the other action. The general problem of our study is to determine the cohomogeneity one actions on a given Riemannian manifold.

Let $M$ be a connected irreducible Riemannian symmetric space of noncompact type. It is known that any such action on $M$ either induces a foliation on $M$ or has exactly one singular orbit [1, Proposition 1]. This induces a disjoint union of the moduli space of cohomogeneity one actions on $M, \mathfrak{M}=\mathfrak{M}_{F} \cup \mathfrak{M}_{S}$, where $\mathfrak{M}_{F}$ is the set of all homogeneous codimension one foliations on $M$ modulo isometric congruence and $\mathfrak{M}_{S}$ is the set of all cohomogeneity one actions on $M$ with a singular orbit $F$ modulo orbit equivalence. In [2] we derived a complete description of the moduli space $\mathfrak{M}_{F}$. In this case, cohomogeneity one actions are orbit equivalent if and only if the induced foliations are isometric congruent.

Low-dimensional singular orbits of cohomogeneity one actions on Riemannian manifolds $M$ are necessarily totally geodesic. More precisely, if the dimension of a singular orbit is less than $(1 / 2)(\operatorname{dim} M-1)$, then it is totally geodesic [1]. This motivates to begin the investigation of singular orbits by concentrating first on totally geodesic singular orbits. In this paper, we determine the subset $\mathfrak{M}_{S}^{\text {tg }} \subset \mathfrak{M}_{S}$ of equivalent classes of cohomogeneity one actions on $M$ with totally geodesic singular orbits when $M$ is a connected irreducible Riemannian symmetric space of noncompact type. It is enough to classify totally geodesic submanifolds $F$ in $M$ which arise as the singular orbits of cohomogeneity one actions, since $F$ determine the cohomogeneity one actions up to orbit equivalence. In fact, given a singular orbit $F$ of an isometric cohomogeneity one action on $M$, the other orbits are then just the tubes of different radii around $F$.

2000 Mathematics Subject Classification. Primary 53C35; Secondary 57S20. 
A totally geodesic submanifold $F$ of $M$ is reflective if the geodesic reflection in $F$ is a globally well-defined isometry of $M$. Reflective submanifolds always arise in pairs $\left(F, F^{\perp}\right)$, where $F^{\perp}$ is also a reflective submanifold of $M$ with the property that it is tangent to any normal space of $F$ via some suitable isometry of $M$. The main result of this paper is stated as follows.

THEOREM. Let $M$ be a connected irreducible Riemannian symmetric space of noncompact type and $F$ a totally geodesic submanifold of $M$. Then $F$ arises as the singular orbit of a cohomonegeity one action on $M$ if and only if $F$ is a reflective submanifold of $M$ for which the rank of $F^{\perp}$ is one, or if $F$ is one of the following totally geodesic non-reflective submanifolds:

(1) $F=G_{2}^{2} / S O(4) \subset M=G_{3}^{*}\left(\boldsymbol{R}^{7}\right)=S O^{o}(3,4) / S O(3) S O(4)$,

(2) $F=G_{2}^{\boldsymbol{C}} / G_{2} \subset M=S O(7, C) / S O(7)$,

(3) $F=C^{2} \subset M=G_{2}^{2} / S O(4)$,

(4) $F=S L(3, \boldsymbol{R}) / S O(3) \subset M=G_{2}^{2} / S O(4)$,

(5) $\quad F=S L(3, C) / S U(3) \subset M=G_{2}^{C} / G_{2}$.

It is quite remarkable that all non-reflective examples are related to the exceptional Lie algebra $\mathfrak{g}_{2}$. A complete list of the reflective submanifolds $F$ of $M$ for which $F^{\perp}$ has rank one can be found in Section 3. A look at this list exhibits the following facts. The set $\mathfrak{M}_{S}^{\mathrm{tg}}$ is empty for the exceptional symmetric spaces of $E_{7}^{C}$ and $E_{8}^{C}$ and all their noncompact real forms, and of $E_{6}^{C}$ and its split real form. For all other symmetric spaces, which include all classical symmetric spaces, $\mathfrak{M}_{S}^{\text {tg }}$ is nonempty and finite. From the above theorem we deduce that \# $\mathfrak{M}_{S}^{\text {tg }}=n>3$ only for the hyperbolic spaces $\boldsymbol{R} H^{n+1}, \boldsymbol{C} H^{n-1}$ and $\boldsymbol{H} H^{n-1}$. For the symmetric spaces $\boldsymbol{R} H^{4}, \boldsymbol{C} H^{2}, \boldsymbol{H} H^{2}, \boldsymbol{O} H^{2}, G_{3}^{*}\left(\boldsymbol{R}^{7}\right), G_{2}^{*}\left(\boldsymbol{R}^{2 n}\right)(n \geq 3)$ and $G_{2}^{*}\left(\boldsymbol{C}^{2 n}\right)(n \geq 3)$, we have $\# \mathfrak{M}_{S}^{\mathrm{tg}}=3$. For the symmetric spaces $\boldsymbol{R} H^{3}, G_{k}^{*}\left(\boldsymbol{R}^{n}\right)(1<k<n-k,(k, n) \neq$ $(3,7),(2,2 m), m>2), G_{3}^{*}\left(\boldsymbol{R}^{6}\right), G_{k}^{*}\left(\boldsymbol{C}^{n}\right)(1<k<n-k,(k, n) \neq(2,2 m), m>2), G_{k}^{*}\left(\boldsymbol{H}^{n}\right)$ $(1<k<n-k), S L(3, \boldsymbol{H}) / \operatorname{Sp}(3), S L(3, \boldsymbol{C}) / S U(3), S L(4, \boldsymbol{C}) / S U(4)=S O(6, \boldsymbol{C}) / S O(6)$, $S O(7, C) / S O(7), G_{2}^{2} / S O(4)$ and $E_{6}^{-24} / F_{4}$, we have $\# \mathfrak{M}_{S}^{\text {tg }}=2$. In the remaining cases we have $\# \mathfrak{M}_{S}^{\text {tg }}=1$.

We finally mention that, by a classical result of E. Cartan [3], $\mathfrak{M}_{S}=\mathfrak{M}_{S}^{\text {tg }}$ for the real hyperbolic space $\boldsymbol{R} H^{n}$. However, as was shown in [1], $\mathfrak{M}_{S}^{\text {tg }}$ is strictly contained in $\mathfrak{M}_{S}$ for the other hyperbolic spaces $\boldsymbol{C} H^{n}, \boldsymbol{H} H^{n}$ and $\boldsymbol{O} H^{2}$. The problem to determine $\mathfrak{M}_{S} \backslash \mathfrak{M}_{S}^{\text {tg }}$ is still open for these hyperbolic spaces, as well as for the symmetric spaces of higher rank.

The paper is organized as follows. In Section 2 we explain a duality between totally geodesic singular orbits of cohomogeneity one actions on Riemannian symmetric spaces of noncompact type and of those on Riemannian symmetric spaces of compact type, respectively. We also relate reflective singular orbits to a certain type of action. In Section 3 we classify the reflective submanifolds of irreducible Riemannian symmetric spaces of noncompact type which arise as a singular orbit of a cohomogeneity one action on the symmetric 
space. In Section 4 we derive the analogous classification for totally geodesic non-reflective submanifolds.

We are grateful for the financial support we received from the University of Hull Research Support Fund. We thank the referee for reading carefully the original version of the manuscript and for the suggestions for improvement.

2. Duality. In this section we describe a duality between the totally geodesic singular orbits of cohomogeneity one actions on a noncompact Riemannian symmetric space and those on its dual simply connected compact Riemannian symmetric space. Let $M=G / K$ be a connected Riemannian symmetric space of noncompact type, where $G=I^{o}(M)$ is the connected component of the full isometry group $I(M)$ of $M$ and $K$ is the isotropy subgroup of $G$ at some point $o \in M$. It is known that $G$ is a noncompact semisimple real Lie group and $K$ is a maximal compact subgroup of $G$. Moreover, since every Riemannian symmetric space of noncompact type is simply connected, $K$ is connected. Let $\mathfrak{g}$ and $\mathfrak{k}$ be the Lie algebra of $G$ and $K$, respectively, and $\mathfrak{g}=\mathfrak{k} \oplus \mathfrak{p}$ the corresponding Cartan decomposition of $\mathfrak{g}$. We identify $\mathfrak{p}$ with the tangent space $T_{o} M$ of $M$ at $o$ in the usual way. Let $\mathfrak{g}^{C}$ be the complexification of $\mathfrak{g}$ and $\mathfrak{g}^{*}=\mathfrak{k} \oplus i \mathfrak{p} \subset \mathfrak{g}^{C}$. Then $\mathfrak{g}^{*}$ is a compact real form of $\mathfrak{g}^{C}$. The connected, simply connected Riemannian symmetric space $M^{*}$ associated with the pair $\left(\mathfrak{g}^{*}, \mathfrak{k}\right)$ is called the compact dual space of $M$. Note that $M^{*}$ can be represented as $M^{*}=G^{*} / K$, where $G^{*}$ is the connected, simply connected Lie group with Lie algebra $\mathfrak{g}^{*}$. We denote by $o^{*}$ the corresponding origin in $M^{*}$.

Now, let $F$ be a totally geodesic singular orbit of a cohomogeneity one action on $M$. We may assume that $o \in F$. Let $T_{o} F$ be the tangent space of $F$ at $o$, which we consider as a subspace of $\mathfrak{p} \cong T_{o} M$. Since $F$ is totally geodesic in $M$, the tangent space $T_{o} F$ is a Lie triple system in $\mathfrak{p}$, that is, $\left[\left[T_{o} F, T_{o} F\right], T_{O} F\right] \subset T_{o} F$. We denote by $N_{\mathfrak{k}}\left(T_{O} F\right)$ the normalizer of $T_{o} F$ in $\mathfrak{k}$. As $T_{o} F$ is a Lie triple system in $\mathfrak{p}$, it is easy to see that $\mathfrak{h}=N_{\mathfrak{k}}\left(T_{o} F\right) \oplus T_{o} F$ is a Lie subalgebra of $\mathfrak{g}$. Let $H$ be the connected closed subgroup of $G$ with Lie algebra $\mathfrak{h}$. We claim that $H$ acts on $M$ with cohomogeneity one and a singular orbit $F=H \cdot o$.

By construction, $\mathfrak{h}$ is invariant under the Cartan involution on $\mathfrak{g}$ with respect to the Cartan decomposition $\mathfrak{g}=\mathfrak{k} \oplus \mathfrak{p}$. This implies that the orbit $H \cdot o$ of $H$ through $o$ is totally geodesic in $M$. Moreover, the tangent space of $H \cdot o$ at $o$ is $T_{o} F$ by construction of $\mathfrak{h}$. Both $F$ and $H \cdot o$ are connected, complete, totally geodesic submanifolds of $M$ with the same tangent space at $o$, and hence they must coincide.

Let $k \in K$ be an isometry that leaves $F$ invariant, that is, $k(F)=F$. Then we have $\operatorname{Ad}(k) T_{o} F=k_{* o} T_{o} F=T_{o} F$, where $k_{* o}$ denotes the differential of $k$ at $o$, and hence $k \in$ $N_{K}\left(T_{o} F\right)$, the normalizer of $T_{o} F$ in $K$. By assumption there exists a connected Lie subgroup $H^{\prime}$ of $G$ acting on $M$ with cohomogeneity one such that $F=H^{\prime} \cdot o=H^{\prime} / H_{o}^{\prime}$. The above argument shows that the slice representation of $H_{o}^{\prime}$ on the normal space $v_{o} F$ of $F$ at $o$ is just the restriction of the slice representation of $N_{K}\left(T_{o} F\right)$ on $v_{o} F$. Since by assumption $H_{o}^{\prime}$ acts transitively on the unit sphere in $v_{o} F, N_{K}\left(T_{o} F\right)$ must also act transitively on the unit sphere in $v_{o} F$. We conclude that $H$ acts on $M$ with cohomogeneity one and $F=H \cdot o$. 
Since $T_{o} F$ is a Lie triple system in $\mathfrak{p}$, the linear subspace $i T_{o} F$ is a Lie triple system in $i \mathfrak{p}$. Thus there exists a unique connected, complete, totally geodesic submanifold $F^{*}$ of $M^{*}$ with $o^{*} \in F^{*}$ and $T_{o^{*}} F^{*}=i T_{o} F$. Let $H^{*}$ be the connected Lie subgroup of $G^{*}$ with Lie algebra $\mathfrak{h}^{*}=N_{\mathfrak{k}}\left(T_{o} F\right) \oplus i T_{o} F \subset \mathfrak{g}^{*}$. As above, we see that the orbit $H^{*} \cdot o^{*}$ of $H^{*}$ through $o^{*}$ coincides with $F^{*}$. Moreover, by construction, the slice representations of $N_{K}\left(T_{o} F\right)$ on $v_{o} F$ and on $v_{o^{*}} F^{*}$ are equivalent. Thus $H^{*}$ acts on $M^{*}$ with cohomogeneity one and $F^{*}=$ $H^{*} \cdot o^{*}$ is a totally geodesic singular orbit of this action. Of course, the construction described above can also be done in the other direction, starting from a Riemannian symmetric space of compact type. We summarize this in

PROPOSITION 2.1. Let $F$ be a totally geodesic singular orbit of a cohomogeneity one action on $M$ and $H$ be the connected Lie subgroup of $G$ with Lie algebra $\mathfrak{h}=N_{\mathfrak{k}}\left(T_{o} F\right) \oplus T_{o} F$. Then $H$ acts on $M$ with cohomogeneity one such that $F=H \cdot o$. Moreover, let $H^{*}$ be the connected Lie subgroup of $G^{*}$ with Lie algebra $\mathfrak{h}^{*}=N_{\mathfrak{k}}\left(T_{o} F\right) \oplus i T_{o} F \subset \mathfrak{g}^{*}$. Then $H^{*}$ acts on $M^{*}$ with cohomogeneity one such that $F^{*}=H^{*} \cdot o^{*}$ is a totally geodesic singular orbit of this action.

Conversely, let $F^{*}$ be a totally geodesic singular orbit of a cohomogeneity one action on $M^{*}$ and $H^{*}$ be the connected Lie subgroup of $G^{*}$ with Lie algebra $\mathfrak{h}^{*}=N_{\mathfrak{k}}\left(T_{o^{*}} F^{*}\right) \oplus i T_{o^{*}} F^{*}$. Then $H^{*}$ acts on $M^{*}$ with cohomogeneity one such that $F^{*}=H^{*} \cdot o^{*}$. Moreover, let $H$ be the connected Lie subgroup of $G$ with Lie algebra $\mathfrak{h}=N_{\mathfrak{k}}\left(T_{o^{*}} F^{*}\right) \oplus T_{o^{*}} F^{*} \subset \mathfrak{g}$. Then $H$ acts on $M$ with cohomogeneity one such that $F=H \cdot o$ is a totally geodesic singular orbit of this action.

Thus we see that there is a one-to-one correspondence between the congruence classes of totally geodesic singular orbits of cohomogeneity one actions on $M$ and those of totally geodesic singular orbits of cohomogeneity one actions on the dual space $M^{*}$. We emphasize that this does not yield a one-to-one correspondence between cohomogeneity one actions with a totally geodesic singular orbit on $M$ and those on $M^{*}$. The reason is that a cohomogeneity one action on $M$ has at most one singular orbit, whereas a cohomogeneity one action on $M^{*}$ has exactly two singular orbits. Indeed, if the action on $M^{*}$ has two non-congruent totally geodesic singular orbits, then the above construction gives two non-conjugate cohomogeneity one actions on $M$. For example, let $M^{*}=S^{n}=S O(n+1) / S O(n)$ and $M=\boldsymbol{R} H^{n}=S O^{o}(1, n) / S O(n)$. The action of $H^{*}=S O(k+1) S O(n-k) \subset S O(n+1)$ on $S^{n}$ is of cohomogeneity one with two totally geodesic singular orbits $S^{k}$ and $S^{n-k-1}$. If we choose $F^{*}=S^{k}$, then the corresponding cohomogeneity one action on $\boldsymbol{R} H^{n}$ is given by $H=S O^{o}(1, k) S O(n-k)$ with $F=\boldsymbol{R} H^{k}$ as a totally geodesic singular orbit. But if we choose $F^{*}=S^{n-k-1}$, then $H=S O^{o}(1, n-k-1) S O(k+1)$ with $F=\boldsymbol{R} H^{n-k-1}$ as a totally geodesic singular orbit.

The classification of cohomogeneity one actions on connected, simply connected, irreducible Riemannian symmetric spaces of compact type has been obtained by Hsiang and Lawson (for spheres), Takagi, Uchida and Iwata (for other rank one spaces), and Kollross (for higher rank cases, see [6] for details). An obvious method for obtaining now the classification 
of cohomogeneity one actions on $M$ with a totally geodesic singular orbit would be to determine the totally geodesic singular orbits of the cohomogeneity one actions on $M^{*}$ in the list provided by Kollross. Nevertheless, this is a tedious task which we prefer to avoid as long as possible by employing general theory.

We recall that a connected submanifold $F$ of a Riemannian manifold $M$ is reflective if the geodesic reflection in $F$ is a globally well-defined isometry of $M$, and reflective submanifolds are necessarily totally geodesic. However, note that a totally geodesic submanifold is not always reflective. If $M=G / K$ is a connected, simply connected, Riemannian symmetric space of compact or of noncompact type, there is a simple criterion to decide whether a totally geodesic submanifold $F$ is reflective or not. When $F$ is totally geodesic, then the tangent space $T_{o} F$ of $F$ at any point $o$ is a Lie triple system in the vector space $\mathfrak{p}$ of the corresponding Cartan decomposition of $\mathfrak{g}$. Then $F$ is reflective if and only if the normal space $v_{o} F$ is also a Lie triple system in $\mathfrak{p}$. The simplest example of a non-reflective totally geodesic submanifold is a $k$-dimensional real hyperbolic space $\boldsymbol{R} H^{k}$ canonically embedded in $n$-dimensional complex hyperbolic space $\boldsymbol{C} H^{n}$ as a totally geodesic submanifold for all $k<n$. For $k=n, \boldsymbol{R} H^{n}$ is a reflective submanifold of $\boldsymbol{C} H^{n}$.

The following proposition will be very useful for classification purposes.

PROPOSITION 2.2. Let $F^{*}$ be a totally geodesic singular orbit of a cohomogeneity one action on $M^{*}=G^{*} / K$ by a connected subgroup $H^{*}$ of $G^{*}$. Then $F^{*}$ is reflective if and only if there exists a connected, simply connected Riemannian symmetric space $M^{\prime}=G^{*} / K^{\prime}$ such that the actions of $H^{*}$ and $K^{\prime}$ on $M^{*}$ have the same orbits.

Proof. We first assume that $F^{*}$ is reflective. Let $F^{* \perp}$ be the reflective submanifold of $M^{*}$ with $o^{*} \in F^{* \perp}$ and $T_{o^{*}} F^{* \perp}=v_{o^{*}} F^{*}$. Let $\mathfrak{g}^{*}=\mathfrak{k} \oplus \mathfrak{p}^{*}$ be the Cartan decomposition of $\mathfrak{g}^{*}$ at $o^{*}$. We denote by $\langle\cdot, \cdot\rangle$ the negative of the Killing form of $\mathfrak{g}^{*}$. We decompose $\mathfrak{p}^{*}$ orthogonally into $\mathfrak{p}^{*}=T_{o^{*}} F^{*} \oplus T_{o^{*}} F^{* \perp}$ and define an involution $\sigma^{\prime}$ on $\mathfrak{g}^{*}$ by

$$
\sigma^{\prime} X= \begin{cases}X & \text { if } X \in N_{\mathfrak{k}}\left(T_{o^{*}} F^{*}\right) \oplus T_{O^{*}} F^{*}, \\ -X & \text { if } X \in\left(N_{\mathfrak{k}}\left(T_{O^{*}} F^{*}\right)\right)^{\perp} \oplus T_{O^{*}} F^{* \perp},\end{cases}
$$

where $\left(N_{\mathfrak{k}}\left(T_{o^{*}} F^{*}\right)\right)^{\perp}$ is the orthogonal complement of $N_{\mathfrak{k}}\left(T_{o^{*}} F^{*}\right)$ in $\mathfrak{k}$. Note that we have $N_{\mathfrak{k}}\left(T_{o^{*}} F^{*}\right)=N_{\mathfrak{k}}\left(T_{o^{*}} F^{* \perp}\right)$. Since both $T_{o^{*}} F^{*}$ and $T_{o^{*}} F^{* \perp}$ are Lie triple systems, one can show that $\sigma^{\prime}$ is a Lie algebra automorphism. Indeed, since $T_{O^{*}} F^{* \perp}$ is a Lie triple system, we have

$$
\left[T_{O^{*}} F^{* \perp}, T_{O^{*}} F^{* \perp}\right] \subset N_{\mathfrak{k}}\left(T_{O^{*}} F^{* \perp}\right)=N_{\mathfrak{k}}\left(T_{O^{*}} F^{*}\right),
$$

which implies $\left\langle\left[T_{O^{*}} F^{* \perp}, T_{O^{*}} F^{* \perp}\right],\left(N_{\mathfrak{k}}\left(T_{O^{*}} F^{*}\right)^{\perp}\right)\right\rangle=0$ and hence $\left[\left(N_{\mathfrak{k}}\left(T_{O^{*}} F^{*}\right)^{\perp}\right), T_{O^{*}} F^{* \perp}\right]$ $\subset T_{O^{*}} F^{*}$ by means of the Jacobi identity. The other relevant inclusions can be deduced in a similar fashion. Thus $\sigma^{\prime}$ is an involutive Lie algebra automorphism of $\mathfrak{g}^{*}$ and hence induces a Cartan decomposition $\mathfrak{g}^{*}=\mathfrak{k}^{\prime} \oplus \mathfrak{p}^{\prime}$ with $\mathfrak{k}^{\prime}=N_{\mathfrak{k}}\left(T_{o^{*}} F^{*}\right) \oplus T_{o^{*}} F^{*}$ and $\mathfrak{p}^{\prime}=$ $\left(N_{\mathfrak{k}}\left(T_{O^{*}} F^{*}\right)\right)^{\perp} \oplus T_{0^{*}} F^{* \perp}$. Let $K^{\prime}$ be the connected closed subgroup of $G^{*}$ with Lie algebra $\mathfrak{k}^{\prime}$. Then $G^{*} / K^{\prime}$ is a connected, simply connected Riemannian symmetric space of compact 
type. By construction, the orbit $K^{\prime} \cdot o^{*}$ of $K^{\prime}$ through $o^{*} \in M^{*}$ coincides with the reflective submanifold $F^{*}$. Since the Lie algebra $\mathfrak{h}^{*}$ of $H^{*}$ is contained in $\mathfrak{k}^{\prime}$ and $H^{*}$ acts on $M^{*}$ with cohomogeneity one, the cohomogeneity of the action of $K^{\prime}$ on $M^{*}$ must also be one. This implies that the orbits of the actions of $H^{*}$ and $K^{\prime}$ on $M^{*}$ coincide.

Conversely, assume that there exists a connected, simply connected Riemannian symmetric space $M^{\prime}=G^{*} / K^{\prime}$ such that the actions of $H^{*}$ and $K^{\prime}$ on $M^{*}$ have the same orbits. Let $\sigma^{*}$ and $\sigma^{\prime}$ be the Cartan involutions of $\mathfrak{g}^{*}$ with respect to $\mathfrak{k}$ and $\mathfrak{k}^{\prime}$, respectively, and $\mathfrak{g}^{*}=\mathfrak{k} \oplus \mathfrak{p}^{*}$ be the Cartan decomposition of $\mathfrak{g}^{*}$ with respect to $\sigma^{*}$. Since $F^{*}$ is totally geodesic, it follows by a result of Hermann [5] that $\sigma^{*}$ and $\sigma^{\prime}$ commute. Thus $\sigma^{\prime}$ induces an orthogonal decomposition

$$
\mathfrak{g}^{*}=\left(\mathfrak{k}_{+} \oplus \mathfrak{k}_{-}\right) \oplus\left(\mathfrak{p}_{+}^{*} \oplus \mathfrak{p}_{-}^{*}\right),
$$

where the indices \pm indicate the intersections of $\mathfrak{k}$ and $\mathfrak{p}$ with the \pm 1 -eigenspaces of $\sigma^{\prime}$, respectively. By construction, we have $\mathfrak{p}_{+}^{*}=T_{O^{*}} F^{*}$ and $\mathfrak{p}_{-}^{*}=v_{o^{*}} F^{*}$. Standard properties of Cartan decompositions imply $\left[\mathfrak{p}_{-}^{*}, \mathfrak{p}_{-}^{*}\right] \subset \mathfrak{k}_{+}$and $\left[\mathfrak{k}_{+}, \mathfrak{p}_{-}^{*}\right] \subset \mathfrak{p}_{-}^{*}$, which shows that $\mathfrak{p}_{-}^{*}$ is a Lie triple system in $\mathfrak{p}^{*}$. Thus $F^{*}$ is a reflective submanifold of $M^{*}$.

If $G^{*} / K$ and $G^{*} / K^{\prime}$ are two connected, simply connected Riemannian symmetric spaces of compact type, then the action of each isotropy group on the other symmetric space is often called a Hermann action. Hermann proved that such an action is variationally complete in the sense of Bott and Samelson. Proposition 2.2 thus says that a totally geodesic singular orbit of a cohomogeneity one action on a connected, simply connected Riemannian symmetric space is reflective if and only if it is a totally geodesic orbit of a Hermann action.

In the next section we will investigate which reflective submanifolds can be the singular orbit of a cohomogeneity one action.

3. Reflective singular orbits. The totally geodesic singular orbits of cohomogeneity one actions on the rank one symmetric spaces of noncompact type, that is, on the hyperbolic spaces $\boldsymbol{R} H^{n}, \boldsymbol{C} H^{n}, \boldsymbol{H} H^{n}$ and $\boldsymbol{O} H^{2}$, have been classified by the first author and Brück in [1] as follows.

THEOREM 3.1 ([1]). A totally geodesic submanifold of $M \in\left\{\boldsymbol{R} H^{n}, \boldsymbol{C} H^{n}, \boldsymbol{H} H^{n}, \boldsymbol{O} H^{2}\right\}$, $n>1$, is the singular orbit of a cohomogeneity one action on $M$ if and only if it is one of the following reflective submanifolds:

$$
\begin{aligned}
\boldsymbol{R} H^{n} & : \text { pt }, \boldsymbol{R} H^{1}, \ldots, \boldsymbol{R} H^{n-2}, \\
\boldsymbol{C} H^{n} & : \text { pt }, \boldsymbol{C} H^{1}, \ldots, \boldsymbol{C} H^{n-1}, \boldsymbol{R} H^{n}, \\
\boldsymbol{H} H^{n} & : \text { pt }, \boldsymbol{H} H^{1}, \ldots, \boldsymbol{H} H^{n-1}, \boldsymbol{C} H^{n}, \\
\boldsymbol{O} H^{2} & : \text { pt }, \boldsymbol{O} H^{1}, \boldsymbol{H} H^{2} .
\end{aligned}
$$

Here, pt means a point, which arises as a singular orbit of the action of the isotropy group $K$. For rank one symmetric spaces the action of the isotropy group is obviously of cohomogeneity one. All submanifolds in the above list are reflective submanifolds. Indeed, it follows from this classification that a totally geodesic submanifold $F$ in one of these hyperbolic spaces 
is a singular orbit of a cohomogeneity one action on that space if and only if $F$ is reflective. We thus have only to deal with the case of higher ranks, that is, rank greater than one.

We keep the notation introduced in Section 2. Let $F$ be a reflective submanifold of $M$ and $F^{\perp}$ be its complement at $o \in F$, that is, the connected, complete, totally geodesic submanifold of $M$ with $T_{o} F^{\perp}=v_{o} F$. Note that $F^{\perp}$ is reflective as well. We recall that a connected, complete, totally geodesic submanifold of a Riemannian symmetric space is itself a Riemannian symmetric space. The restrictions of the geodesic symmetries of the ambient space to the submanifold provide the geodesic symmetries. The following result yields a simple criterion for deciding whether a reflective submanifold arises as a singular orbit of a cohomogeneity one action.

PROPOSITION 3.2. Let $F$ be a reflective submanifold of a connected Riemannian symmetric space $M$ of noncompact type. Then $F$ is a singular orbit of a cohomogeneity one action on $M$ if and only if the rank of $F^{\perp}$ is one.

PROOF. We can assume that $M$ has rank greater than one, since the rank one case has been settled by Theorem 3.1.

First assume that $F$ is a singular orbit of a cohomogeneity one action on $M$. We may assume that $o \in F$. Then there exists a Lie subgroup $H$ of $G$ acting on $M$ with cohomogeneity one and with $F=H \cdot o=H / H_{o}$. By assumption, the slice representation of $H_{o}$ on the normal space $v_{o} F$ is transitive on the unit sphere in $v_{o} F$. Since $F^{\perp}$ is totally geodesic, any isometry in $H_{o}$ restricts to an isometry of $F^{\perp}$. This shows that the slice representation of $H_{o}$ on $v_{o} F$ is the restriction to $H_{o}$ of the isotropy representation of $F^{\perp}$ on $T_{o} F^{\perp}$. It follows that the isotropy subgroup of $F^{\perp}$ is also transitive on the unit sphere in $T_{o} F^{\perp}$, and hence $F^{\perp}$ is a rank one symmetric space.

Conversely, assume that the rank of $F^{\perp}$ is one. We define a linear subspace $\mathfrak{h}$ of $\mathfrak{g}$ by $\mathfrak{h}=N_{\mathfrak{k}}\left(T_{o} F\right) \oplus T_{o} F \subset \mathfrak{k} \oplus \mathfrak{p}$. Since $T_{o} F$ is a Lie triple system in $\mathfrak{p}$, the subspace $\mathfrak{h}$ is a Lie subalgebra of $\mathfrak{g}$. Let $H$ be the connected closed subgroup of $G$ with Lie algebra $\mathfrak{h}$. Then, as shown above, we have $H \cdot o=F$. Let $k$ be an isometry of $F^{\perp}$ in the identity component of the isometry group of $F^{\perp}$ and assume that $k$ fixes $o$. Since $M$ has rank greater than one, we must have $\operatorname{dim} F^{\perp}>1$. The assumption that $F^{\perp}$ has rank one now implies that it is semisimple. Thus $k$ is generated by some curvature transformation $R^{F^{\perp}}(X, Y): T_{o} F^{\perp} \rightarrow$ $T_{o} F^{\perp}, X, Y \in T_{o} F^{\perp}$. This is because the isotropy algebra coincides with the holonomy algebra for semisimple Riemannian symmetric spaces. Since $F^{\perp}$ is totally geodesic in $M$, $R^{F^{\perp}}(X, Y)$ is just the restriction of the curvature transformation $R^{M}(X, Y): T_{o} M \rightarrow T_{o} M$ to $T_{o} F^{\perp}$. Since $M$ is also semisimple by assumption, it follows that $k$ extends to an isometry $k^{\prime}$ of $M$. Then $\operatorname{Ad}\left(k^{\prime}\right)$ normalizes $T_{o} F$, and the isotropy representation of $F^{\perp}$ in $o$ is a restriction of the slice representation of $H_{o}$ on $v_{o} F=T_{o} F^{\perp}$. Since $F^{\perp}$ has rank one, we eventually conclude that $H_{o}$ acts transitively on the unit sphere in $v_{o} F$, which means that $H$ acts with cohomogeneity one on $M$. 
Leung classified in [7] and [8] the reflective submanifolds of connected, simply connected, irreducible Riemannian symmetric spaces of compact type. Using duality and Proposition 3.2, we now obtain the classification of reflective submanifolds of connected, irreducible Riemannian symmetric spaces of noncompact type which arise as a singular orbit of a cohomogeneity one action. We use the following notation for hyperbolic Grassmann manifolds: $G_{k}^{*}\left(\boldsymbol{R}^{n}\right)=S O^{o}(k, n-k) / S O(k) S O(n-k), G_{k}^{*}\left(\boldsymbol{C}^{n}\right)=S U(k, n-k) / S(U(k) U(n-k))$ and $G_{k}^{*}\left(\boldsymbol{H}^{n}\right)=\operatorname{Sp}(k, n-k) / \operatorname{Sp}(k) \operatorname{Sp}(n-k)$.

THEOREM 3.3. Let $M$ be a connected, irreducible Riemannian symmetric space of noncompact type and rank greater than one. Let $F$ be a reflective submanifold of $M$. Then $F$ is the singular orbit of a cohomogeneity one action on $M$ if and only if $F$ is one of the following reflective submanifolds:

$$
\begin{aligned}
& G_{k}^{*}\left(\boldsymbol{R}^{n}\right)(1<k<n-k,(k, n) \neq(2,2 m), m>2): G_{k-1}^{*}\left(\boldsymbol{R}^{n-1}\right), G_{k}^{*}\left(\boldsymbol{R}^{n-1}\right), \\
& G_{k}^{*}\left(\boldsymbol{R}^{2 k}\right)(k \geq 4): G_{k-1}^{*}\left(\boldsymbol{R}^{2 k-1}\right)=G_{k}^{*}\left(\boldsymbol{R}^{2 k-1}\right), \\
& G_{2}^{*}\left(\boldsymbol{R}^{2 n}\right)(n \geq 3): G_{1}^{*}\left(\boldsymbol{R}^{2 n-1}\right)=\boldsymbol{R} H^{2 n-2}, G_{2}^{*}\left(\boldsymbol{R}^{2 n-1}\right), G_{1}^{*}\left(\boldsymbol{C}^{n}\right)=\boldsymbol{C} H^{n-1}, \\
& G_{3}^{*}\left(\boldsymbol{R}^{6}\right)=S L(4, \boldsymbol{R}) / S O(4): G_{2}^{*}\left(\boldsymbol{R}^{5}\right)=G_{3}^{*}\left(\boldsymbol{R}^{5}\right), S L(3, \boldsymbol{R}) / S O(3) \times \boldsymbol{R}, \\
& G_{k}^{*}\left(\boldsymbol{C}^{n}\right)(1<k<n-k,(k, n) \neq(2,2 m), m>2): G_{k-1}^{*}\left(\boldsymbol{C}^{n-1}\right), G_{k}^{*}\left(\boldsymbol{C}^{n-1}\right) \text {, } \\
& G_{k}^{*}\left(\boldsymbol{C}^{2 k}\right)(k \geq 3): G_{k-1}^{*}\left(\boldsymbol{C}^{2 k-1}\right)=G_{k}^{*}\left(\boldsymbol{C}^{2 k-1}\right), \\
& G_{2}^{*}\left(\boldsymbol{C}^{2 n}\right)(n \geq 3): G_{1}^{*}\left(\boldsymbol{C}^{2 n-1}\right)=\boldsymbol{C} H^{2 n-2}, G_{2}^{*}\left(\boldsymbol{C}^{2 n-1}\right), G_{1}^{*}\left(\boldsymbol{H}^{n}\right)=\boldsymbol{H} H^{n-1} \text {, } \\
& G_{k}^{*}\left(\boldsymbol{H}^{n}\right)(1<k<n-k): G_{k-1}^{*}\left(\boldsymbol{H}^{n-1}\right), G_{k}^{*}\left(\boldsymbol{H}^{n-1}\right), \\
& G_{k}^{*}\left(\boldsymbol{H}^{2 k}\right)(k \geq 2): G_{k-1}^{*}\left(\boldsymbol{H}^{2 k-1}\right)=G_{k}^{*}\left(\boldsymbol{H}^{2 k-1}\right) \text {, } \\
& S L(n, \boldsymbol{R}) / S O(n)(n=3 \text { or } n \geq 5): S L(n-1, \boldsymbol{R}) / S O(n-1) \times \boldsymbol{R} \text {, } \\
& S L(n, \boldsymbol{H}) / S p(n)(n \geq 4): S L(n-1, \boldsymbol{H}) / S p(n-1) \times \boldsymbol{R}, \\
& S L(3, \boldsymbol{H}) / \operatorname{Sp}(3): S L(2, \boldsymbol{H}) / \operatorname{Sp}(2) \times \boldsymbol{R}=\boldsymbol{R} H^{5} \times \boldsymbol{R}, S L(3, \boldsymbol{C}) / S U(3) \text {, } \\
& S O(n, \boldsymbol{H}) / U(n)(n \geq 5): S O(n-1, \boldsymbol{H}) / U(n-1) \text {, } \\
& \operatorname{Sp}(n, \boldsymbol{R}) / U(n)(n \geq 3): S p(n-1, \boldsymbol{R}) / U(n-1) \times \boldsymbol{R} H^{2}, \\
& S L(n, \boldsymbol{C}) / S U(n)(n \geq 5): S L(n-1, \boldsymbol{C}) / S U(n-1) \times \boldsymbol{R} \text {, } \\
& S L(4, \boldsymbol{C}) / S U(4)=S O(6, \boldsymbol{C}) / S O(6): S L(3, \boldsymbol{C}) / S U(3) \times \boldsymbol{R}, S O(5, \boldsymbol{C}) / S O(5) \text {, } \\
& S L(3, \boldsymbol{C}) / S U(3): S L(2, \boldsymbol{C}) / S U(2) \times \boldsymbol{R}=\boldsymbol{R} H^{3} \times \boldsymbol{R}, S L(3, \boldsymbol{R}) / S O(3), \\
& S O(n, C) / S O(n)(n=5 \text { or } n \geq 7): S O(n-1, C) / S O(n-1) \text {, } \\
& \operatorname{Sp}(n, \boldsymbol{C}) / \operatorname{Sp}(n)(n \geq 3): \operatorname{Sp}(n-1, \boldsymbol{C}) / \operatorname{Sp}(n-1) \times \operatorname{Sp}(1, \boldsymbol{C}) / \operatorname{Sp}(1), \\
& E_{6}^{2} / S U(6) S U(2): F_{4}^{4} / S p(3) S U(2) \text {, } \\
& E_{6}^{-14} / \operatorname{Spin}(10) S O(2): \boldsymbol{O} H^{2} \text {, } \\
& E_{6}^{-24} / F_{4}: \boldsymbol{R} H^{9} \times \boldsymbol{R}, S L(3, \boldsymbol{H}) / S p(3), \\
& F_{4}^{4} / \operatorname{Sp}(3) S U(2): G_{4}^{*}\left(\boldsymbol{R}^{9}\right) \text {, } \\
& F_{4}^{C} / F_{4}: S O(9, C) / S O(9) \text {. }
\end{aligned}
$$

We finally remark that any two isometric reflective submanifolds in a connected, irreducible Riemannian symmetric space of noncompact type are congruent by an element in the full isometry group (Leung [8]). This implies that from each of the above reflective submanifolds we obtain indeed only one cohomogeneity one action on $M$ up to orbit equivalence. 
4. Non-reflective singular orbits. The classification (up to orbit equivalence) of cohomogeneity one actions on connected irreducible Riemannian symmetric spaces of noncompact type with a reflective singular orbit turned out to be quite simple due to Leung's classification of reflective submanifolds. We will now investigate the case of non-reflective singular orbits. In this case it is a problem whether such actions exist. It follows from Theorem 3.1 that any totally geodesic orbit of a cohomogeneity one action must be reflective if the rank of $M$ is one. In this section we will show that this is no longer true if the rank is greater than one.

We start with a lemma useful for the case when $M^{*}$ is a compact Lie group.

LEMMA 4.1. Let $G^{*}$ be a connected, simply connected, compact Lie group and $H_{1}^{*}, H_{2}^{*}$ be connected maximal subgroups of $G^{*}$. Consider the action of $H_{1}^{*} \times H_{2}^{*}$ on $G^{*}$ defined by $\left(h_{1}, h_{2}\right) \cdot g=h_{1} g h_{2}^{-1}$ for all $h_{1} \in H_{1}^{*}, h_{2} \in H_{2}^{*}$ and $g \in G^{*}$. Assume that this action is of cohomogeneity one and that an orbit $\left(H_{1}^{*} \times H_{2}^{*}\right) \cdot g$ through $g \in G^{*}$ is a totally geodesic singular orbit. Then $H_{1}^{*}=H_{2}^{*}$ and $g$ is in the normalizer $N_{G^{*}}\left(H_{1}^{*}\right)$ of $H_{1}^{*}$ in $G^{*}$.

Proof. Assume that $\left(H_{1}^{*} \times H_{2}^{*}\right) \cdot g$ is a totally geodesic singular orbit. First of all, the stabilizer of $H_{1}^{*} \times H_{2}^{*}$ at $g$ is

$$
H_{1}^{*} \cap g H_{2}^{*} g^{-1}=\left\{\left(g h_{2} g^{-1}, h_{2}\right) \mid h_{2} \in H_{2}^{*}, g h_{2} g^{-1} \in H_{1}^{*}\right\},
$$

and hence

$$
\left(H_{1}^{*} \times H_{2}^{*}\right) \cdot g=\frac{H_{1}^{*} \times H_{2}^{*}}{H_{1}^{*} \cap g H_{2}^{*} g^{-1}} .
$$

As $\left(H_{1}^{*} \times H_{2}^{*}\right) \cdot g$ is a singular orbit, the stabilizer at $g$ must act transitively on a sphere of positive dimension. Moreover, since $\left(H_{1}^{*} \times H_{2}^{*}\right) \cdot g$ is totally geodesic, the singular orbit is also a symmetric space. Altogether this can happen only if $H_{2}^{*}=H_{1}^{*}=g H_{2}^{*} g^{-1}$.

Assume that $H$ acts on $M$ with cohomogeneity one with a totally geodesic non-reflective singular orbit $F$. According to Proposition 2.1, we can then construct a cohomogeneity one action of a subgroup $H^{*} \subset I^{o}\left(M^{*}\right)$ on the simply connected dual space $M^{*}$ with a totally geodesic non-reflective singular orbit $F^{*}$. The action of $H^{*}$ must be orbit equivalent to a cohomogeneity one action in the list provided by Kollross [6]. Because of Theorem 3.1 we may assume that the rank of $M$, and hence of $M^{*}$, is greater than one. Taking into account of Proposition 2.2 and Lemma 4.1 the only possibilities for a cohomogeneity one action on $M^{*}$ with a non-reflective totally geodesic singular orbit are the following:

(a) the action of $S O(2 n-2)$ on $S O(2 n-1) / U(n-1)=S O(2 n) / U(n)(n \geq 4)$,

(b) the action of $G_{2}$ on $S O(7) / S O(3) S O(4)=G_{3}^{+}\left(\boldsymbol{R}^{7}\right)$,

(c) the action of $G_{2}$ on $S O(7) / U(3)=S O(8) / U(4)=S O(8) / S O(2) S O(6)=$ $G_{2}^{+}\left(\boldsymbol{R}^{8}\right)$,

(d) the action of $\operatorname{Spin}(9)$ on $S O(16) / S O(2) S O(14)=G_{2}^{+}\left(\boldsymbol{R}^{16}\right)$,

(e) the action of $\operatorname{Sp}(n) \operatorname{Sp}(1)$ on $S O(4 n) / S O(2) S O(4 n-2)=G_{2}^{+}\left(\boldsymbol{R}^{4 n}\right)(n \geq 2)$,

(f) the action of $S U(3)$ on $G_{2} / S O(4)$,

(g) the action of $G_{2} \times G_{2}$ on $\operatorname{Spin}(7)$,

(h) the action of $S U(3) \times S U(3)$ on $G_{2}$. 
We will now discuss these actions individually.

(a) $S O(2 n-2)$ is contained in $S O(2 n-1)$, and since both groups act with cohomogeneity one on $S O(2 n) / U(n)$, they must be orbit equivalent. According to Proposition 2.2 , every totally geodesic singular orbit of the action of $S O(2 n-1)$ on $S O(2 n) / U(n)$ is reflective, and hence we can ignore this action.

(b) We decompose the Lie algebra $\mathfrak{s o}$ (7) orthogonally into

$$
\mathfrak{s o}(7)=\mathfrak{g}_{2} \oplus \boldsymbol{R}^{7},
$$

where $\boldsymbol{R}^{7}$ is the 7-dimensional irreducible representation of $\mathfrak{g}_{2}$. Let $\mathfrak{h}$ be a Cartan subalgebra of $\mathfrak{g}_{2}$ and

$$
\mathfrak{g}_{2}=\mathfrak{h} \oplus \bigoplus_{\alpha \in \Sigma^{+}} \mathfrak{g}_{\alpha}
$$

be the corresponding root space decomposition of $\mathfrak{g}_{2}$ with

$$
\Sigma^{+}=\left\{\alpha_{1}, \alpha_{2}, \alpha_{1}+\alpha_{2}, 2 \alpha_{1}+\alpha_{2}, 3 \alpha_{1}+\alpha_{2}, 3 \alpha_{1}+2 \alpha_{2}\right\} .
$$

The fundamental weight of the irreducible representation of $\mathfrak{g}_{2}$ on $\boldsymbol{R}^{7}$ is $2 \alpha_{1}+\alpha_{2}$, and the weight space decomposition of $\boldsymbol{R}^{7}$ is

$$
\boldsymbol{R}^{7}=V_{0} \oplus V_{\alpha_{1}} \oplus V_{\alpha_{1}+\alpha_{2}} \oplus V_{2 \alpha_{1}+\alpha_{2}}
$$

where $V_{0}$ is one-dimensional and the other three weight spaces are two-dimensional. Note that $\mathfrak{h} \oplus V_{0}$ is a Cartan subalgebra of $\mathfrak{s o}(7)$, and weight spaces satisfy the general relation $\left[V_{\alpha}, V_{\beta}\right] \subset V_{\alpha \pm \beta} \oplus \mathfrak{g}_{\alpha \pm \beta}$. We now define a linear subspace $\mathfrak{k}$ of $\mathfrak{s o}(7)$ by

$$
\mathfrak{k}=\mathfrak{h} \oplus \mathfrak{g}_{\alpha_{1}} \oplus \mathfrak{g}_{3 \alpha_{1}+2 \alpha_{2}} \oplus V_{0} \oplus V_{\alpha_{1}} .
$$

It follows from the bracket relations for root and weight spaces that $\mathfrak{k}$ is a subalgebra of $\mathfrak{s o}(7)$. We choose a nonzero vector $H \in \mathfrak{h}$ with $\alpha_{1}(H)=0$. Then $\boldsymbol{R} H \oplus \mathfrak{g}_{3 \alpha_{1}+2 \alpha_{2}}$ is an ideal in $\mathfrak{k}$ and is isomorphic to $\mathfrak{s o}(3)$. It is now immediate that $\mathfrak{k}$ is isomorphic to $\mathfrak{s o}(3) \oplus \mathfrak{s o}(4)$. The Cartan decomposition of $\mathfrak{s o}(7)$ with respect to $\mathfrak{k}$ is given by $\mathfrak{s o}(7)=\mathfrak{k} \oplus \mathfrak{p}$ with

$$
\mathfrak{p}=\mathfrak{g}_{\alpha_{2}} \oplus \mathfrak{g}_{\alpha_{1}+\alpha_{2}} \oplus \mathfrak{g}_{2 \alpha_{1}+\alpha_{2}} \oplus \mathfrak{g}_{3 \alpha_{1}+\alpha_{2}} \oplus V_{\alpha_{1}+\alpha_{2}} \oplus V_{2 \alpha_{1}+\alpha_{2}} .
$$

Since $\mathfrak{g}_{2}$ is invariant under the Cartan involution of $\mathfrak{s o}(7)$ with respect to the Cartan decomposition $\mathfrak{s o}(7)=\mathfrak{k} \oplus \mathfrak{p}$, the orbit $F^{*}$ of $G_{2}$ through the origin $o^{*}$ of $M^{*}=S O(7) / S O(3) S O$ (4) is totally geodesic. Moreover, since

$$
\mathfrak{g}_{2} \cap(\mathfrak{s o}(3) \oplus \mathfrak{s o}(4))=\mathfrak{h} \oplus \mathfrak{g}_{\alpha_{1}} \oplus \mathfrak{g}_{3 \alpha_{1}+2 \alpha_{2}}=\mathfrak{s o}(3) \oplus \mathfrak{s o}(3)=\mathfrak{s o}(4),
$$

we have $F^{*}=G_{2} / S O(4)$. The normal space $v_{o^{*}} F^{*}$ of $F^{*}$ at $o^{*}$ is

$$
v_{o^{*}} F^{*}=V_{\alpha_{1}+\alpha_{2}} \oplus V_{2 \alpha_{1}+\alpha_{2}} \text {. }
$$

Note that the action of $\mathfrak{s o}(4)$ on this 4-dimensional space is the standard one, which confirms that the action of $G_{2}$ on $M^{*}$ is indeed of cohomogeneity one. Using again the bracket relations for weight spaces, we get

$$
\left[v_{o^{*}} F^{*}, v_{o^{*}} F^{*}\right] \subset \mathfrak{h} \oplus \mathfrak{g}_{\alpha_{1}} \oplus \mathfrak{g}_{3 \alpha_{1}+2 \alpha_{2}} \oplus V_{0} \oplus V_{\alpha_{1}} .
$$


But

$$
\left[V_{\alpha_{1}}, V_{\alpha_{1}+\alpha_{2}}\right] \subset \mathfrak{g}_{\alpha_{2}} \oplus \mathfrak{g}_{2 \alpha_{1}+\alpha_{2}} \oplus V_{2 \alpha_{1}+\alpha_{2}}
$$

which readily implies that $v_{O^{*}} F^{*}$ is not a Lie triple system in $\mathfrak{p}$. Hence $F^{*}$ is non-reflective. Since $G_{2}$ is connected, it preserves the orientation of 3-planes in $\boldsymbol{R}^{7}$. Therefore the second singular orbit is $\rho^{*}\left(F^{*}\right)$, where $\rho^{*}$ is the natural orientation-reversing isometry of $G_{3}^{+}\left(\boldsymbol{R}^{7}\right)$. Clearly, $\rho^{*}\left(F^{*}\right)$ is another copy of $G_{2} / S O(4)$ embedded in $G_{3}^{+}\left(\boldsymbol{R}^{7}\right)$ as a totally geodesic non-reflective submanifold. Using Proposition 2.1 , from each of the two totally geodesic singular orbits we can now construct a cohomogeneity one action on $M=G_{3}^{*}\left(\boldsymbol{R}^{7}\right)=$ $S O^{o}(3,4) / S O(3) S O(4)$. In both cases the corresponding subgroup of $S O^{o}(3,4)$ is the split real form $G_{2}^{2}$ of $G_{2}^{C}$. The isometry $\rho^{*}$ of $M^{*}$ gives rise to an isometry $\rho$ of $M$ under which the two cohomogeneity one actions on $M$ are conjugate. Thus, to sum up, the action of $G_{2}$ on $G_{3}^{+}\left(\boldsymbol{R}^{7}\right)$ induces exactly one (up to orbit equivalence) cohomogeneity one action on $G_{3}^{*}\left(\boldsymbol{R}^{7}\right)$. The corresponding subgroup of $S O^{\circ}(3,4)$ is $G_{2}^{2}$, and the totally geodesic non-reflective singular orbit is isometric to the noncompact symmetric space $G_{2}^{2} / S O(4)$.

(c) The action of $G_{2}$ on $G_{2}^{+}\left(\boldsymbol{R}^{8}\right)$ can be seen by identifying $\boldsymbol{R}^{8}$ with the octonions $\boldsymbol{O}$ and taking into account that $G_{2}$ is the automorphism group of $\boldsymbol{O}$. The stabilizer of $G_{2}$ at a unit vector $u \in \operatorname{Im} \boldsymbol{O}$ is $S U(3)$, and that of $S U(3)$ at a unit vector $v \in \operatorname{Im} \boldsymbol{O}$ perpendicular to $u$ is $S U$ (2). Thus the stabilizer of $G_{2}$ at the 2-plane $V$ spanned by $u$ and $v$ is $U(2)$, and hence $G_{2} \cdot V=G_{2} / U(2)=G_{2}^{+}\left(\boldsymbol{R}^{7}\right)$. The stabilizer of $G_{2}$ at the 2-plane $U$ spanned by $1 \in \operatorname{Re} \boldsymbol{O}$ and $u$ is $S U(3)$, and hence $G_{2} \cdot U=G_{2} / S U(3)=S^{6}$. These two singular orbits coincide with the singular orbits of the cohomogeneity one action of $S O(7)$ on $G_{2}^{+}\left(\boldsymbol{R}^{8}\right)$, where the $S O(7)$ sits inside $S O(8)$ according to the decomposition $\boldsymbol{R}^{8}=\boldsymbol{R} \oplus \boldsymbol{R}^{7}=\operatorname{Re} \boldsymbol{O} \oplus \operatorname{Im} \boldsymbol{O}$. This implies that the action of $G_{2}$ on $G_{2}^{+}\left(\boldsymbol{R}^{8}\right)$ is orbit equivalent to the action of $S O(7)$ on $G_{2}^{+}\left(\boldsymbol{R}^{8}\right)$, which is an action of the type described in Proposition 2.2. Hence the action of $G_{2}$ on $G_{2}^{+}\left(\boldsymbol{R}^{8}\right)$ has no totally geodesic non-reflective singular orbits.

(d) The stabilizer of $\operatorname{Spin}(9)$ at a unit vector $u \in \boldsymbol{R}^{16}$ is $\operatorname{Spin}(7)$. The representation of this $\operatorname{Spin}(7)$ on $\boldsymbol{R}^{16}$ has three irreducible components $\boldsymbol{R} u \oplus \boldsymbol{R}^{7} \oplus \boldsymbol{R}^{8}$, where the representation on $\boldsymbol{R} u$ is trivial, the one on $\boldsymbol{R}^{7}$ is the standard one, and the one on $\boldsymbol{R}^{8}$ is the spin representation. Take unit vectors $v \in \boldsymbol{R}^{7}$ and $w \in \boldsymbol{R}^{8}$. One can see that the orbit through the 2-plane spanned by $u$ and $v$ (resp. $u$ and $w$ ) is $\operatorname{Spin}(9) / \operatorname{Spin}(6) S O(2)$ (resp. $\operatorname{Spin}(9) / G_{2} S O(2)$ ). A dimension argument shows that they are the singular orbits of the $\operatorname{Spin}(9)$-action on $G_{2}^{+}\left(\boldsymbol{R}^{16}\right)$. Since both singular orbits are not symmetric, they cannot be totally geodesic. Thus we can ignore this action.

(e) The stabilizer of $\operatorname{Sp}(n) \operatorname{Sp}(1)$ at a unit vector $u \in \boldsymbol{R}^{4 n}$ is $\operatorname{Sp}(n-1) \operatorname{Sp}(1)$. The representation of this $\operatorname{Sp}(n-1) \operatorname{Sp}(1)$ on $\boldsymbol{R}^{4 n}$ has three irreducible components $\boldsymbol{R} u \oplus \boldsymbol{R}^{3} \oplus$ $\boldsymbol{R}^{4 n-4}$, where the representation on $\boldsymbol{R} u$ is trivial, the one on $\boldsymbol{R}^{3}$ is equivalent to the standard representation of $S p(1)$ on $\boldsymbol{R}^{3}$, and the one on $\boldsymbol{R}^{4 n-4}$ is the standard representation. Similar to the argument of case (d), one can obtain the singular orbits by taking unit vectors $v \in \boldsymbol{R}^{3}$ and $w \in \boldsymbol{R}^{4 n-4}$. The orbit through the 2-plane spanned by $u$ and $v$ is $\operatorname{Sp}(n) \operatorname{Sp}(1) / \operatorname{Sp}(n-$ 1) $U(1) U(1)=C P^{2 n-1} \times C P^{1}$. This is symmetric, but it follows from the classification 
of totally geodesic submanifolds in real Grassmannians of oriented 2-planes by Chen and Nagano [4] that it cannot be totally geodesic. The other singular orbit is the orbit through the 2-plane spanned by $u$ and $w, S p(n) S p(1) / S p(n-2) U(1) S p(1)=S p(n) / S p(n-2) U(1)$, which is not symmetric. Thus we can ignore this action.

(f) Let $\mathfrak{h}$ be a Cartan subalgebra of $\mathfrak{g}_{2}$ and

$$
\mathfrak{g}_{2}=\mathfrak{h} \oplus \bigoplus_{\alpha \in \Sigma^{+}} \mathfrak{g}_{\alpha}
$$

the corresponding root space decomposition of $\mathfrak{g}_{2}$, where we use the same notation for the roots of $\mathfrak{g}_{2}$ as in case (b). We define two subalgebras of $\mathfrak{g}_{2}$ respectively by

$$
\mathfrak{s o}(4)=\mathfrak{h} \oplus \mathfrak{g}_{\alpha_{1}} \oplus \mathfrak{g}_{3 \alpha_{1}+2 \alpha_{2}}
$$

and

$$
\mathfrak{s u}(3)=\mathfrak{h} \oplus \mathfrak{g}_{\alpha_{2}} \oplus \mathfrak{g}_{3 \alpha_{1}+\alpha_{2}} \oplus \mathfrak{g}_{3 \alpha_{1}+2 \alpha_{2}} .
$$

The first subalgebra gives the Cartan decomposition $\mathfrak{g}_{2}=\mathfrak{s o}(4) \oplus \mathfrak{p}$ with

$$
\mathfrak{p}=\mathfrak{g}_{\alpha_{2}} \oplus \mathfrak{g}_{\alpha_{1}+\alpha_{2}} \oplus \mathfrak{g}_{2 \alpha_{1}+\alpha_{2}} \oplus \mathfrak{g}_{3 \alpha_{1}+\alpha_{2}} .
$$

Since the second subalgebra $\mathfrak{s u}(3)$ is invariant under the Cartan involution of $\mathfrak{g}_{2}$ with respect to $\mathfrak{g}_{2}=\mathfrak{s o}(4) \oplus \mathfrak{p}$, the orbit $S U(3) \cdot o^{*}$ of $S U(3)$ through the origin $o^{*} \in M^{*}=G_{2} / S O(4)$ is totally geodesic. Since

$$
\mathfrak{s u}(3) \cap \mathfrak{s o}(4)=\mathfrak{h} \oplus \mathfrak{g}_{3 \alpha_{1}+2 \alpha_{2}}=\boldsymbol{R} \oplus \mathfrak{s u}(2)=\mathfrak{u}(2),
$$

we see that $S U(3) \cdot o^{*}$ is a complex projective plane $C P^{2}=S U(3) / U(2)$. The tangent and the normal space of $C P^{2}$ at $o^{*}$ is given by

$$
T_{o^{*}} \boldsymbol{C} P^{2}=\mathfrak{g}_{\alpha_{2}} \oplus \mathfrak{g}_{3 \alpha_{1}+\alpha_{2}} \quad \text { and } \quad v_{o^{*}} \boldsymbol{C} P^{2}=\mathfrak{g}_{\alpha_{1}+\alpha_{2}} \oplus \mathfrak{g}_{2 \alpha_{1}+\alpha_{2}},
$$

respectively. The action of $\mathfrak{u}(2)$ on $v_{O^{*}} \boldsymbol{C} P^{2}$ is equivalent to the action of $\mathfrak{u}(2)$ on $\boldsymbol{C}^{2}$, which implies that the slice representation of $U(2)$ is transitive on the unit sphere in $v_{o^{*}} \boldsymbol{C} H^{2}$. This shows that the action of $S U(3)$ on $G_{2} / S O(4)$ is of cohomogeneity one. Finally, the bracket relations for root spaces show that

$$
\left[\mathfrak{g}_{2 \alpha_{1}+\alpha_{2}}, \mathfrak{g}_{\alpha_{1}+\alpha_{2}}\right] \subset \mathfrak{g}_{\alpha_{1}} \oplus \mathfrak{g}_{3 \alpha_{1}+2 \alpha_{2}} \subset \mathfrak{s o}(4),
$$

and taking bracket again with $\mathfrak{g}_{2 \alpha_{1}+\alpha}$ gives a $\mathfrak{g}_{3 \alpha_{1}+\alpha_{2}}$-component, which implies that $v_{o} \boldsymbol{C} P^{2}$ is not a Lie triple system in $\mathfrak{p}$, and hence $C P^{2}$ is non-reflective. We now construct the dual action on $M=G_{2}^{2} / S O(4)$ according to Proposition 2.1. The corresponding group acting on $M$ with cohomogeneity one is $S U(1,2)$, and the totally geodesic non-reflective singular orbit is a complex hyperbolic plane $C H^{2}=S U(1,2) / S(U(1) U(2))$.

We now investigate the second singular orbit of the $S U(3)$-action on $M^{*}=G_{2} / S O$ (4). For this we move the origin of $G_{2} / S O(4)$ to a suitable point. The starting point is now the Cartan decomposition $\mathfrak{g}=\mathfrak{k} \oplus \mathfrak{p}$, where $\mathfrak{g}=\mathfrak{g}_{2}$ and $\mathfrak{k}=\mathfrak{s o}(4)$, and a maximal abelian subspace $\mathfrak{a}$ in $\mathfrak{p}$, which leads to the root space decomposition

$$
\mathfrak{s o}(4)=\mathfrak{k}=\bigoplus_{\alpha \in \Sigma^{+}} \mathfrak{k}_{\alpha} \quad \text { and } \quad \mathfrak{p}=\mathfrak{a} \oplus \bigoplus_{\alpha \in \Sigma^{+}} \mathfrak{p}_{\alpha} .
$$


Then we have

$$
\mathfrak{s u}(3)=\left(\mathfrak{k}_{\alpha_{2}} \oplus \mathfrak{k}_{3 \alpha_{1}+\alpha_{2}} \oplus \mathfrak{k}_{3 \alpha_{1}+2 \alpha_{2}}\right) \oplus\left(\mathfrak{a} \oplus \mathfrak{p}_{\alpha_{2}} \oplus \mathfrak{p}_{3 \alpha_{1}+\alpha_{2}} \oplus \mathfrak{p}_{3 \alpha_{1}+2 \alpha_{2}}\right) .
$$

Since $\mathfrak{s u}(3)$ is invariant under the Cartan involution with respect to $\mathfrak{g}=\mathfrak{k} \oplus \mathfrak{p}$, the orbit $F^{*}$ of $S U$ (3) through the origin $o^{*}$ is totally geodesic in $M^{*}$. Moreover, since

$$
\mathfrak{s u}(3) \cap \mathfrak{s o}(4)=\mathfrak{k}_{\alpha_{2}} \oplus \mathfrak{k}_{3 \alpha_{1}+\alpha_{2}} \oplus \mathfrak{k}_{3 \alpha_{1}+2 \alpha_{2}}=\mathfrak{s o}(3),
$$

the singular orbit $F^{*}$ is isometric to the symmetric space $S U(3) / S O(3)$. The tangent and the normal space of $F^{*}$ at $o^{*}$ is

$$
T_{o^{*}} F^{*}=\mathfrak{a} \oplus \mathfrak{p}_{\alpha_{2}} \oplus \mathfrak{p}_{3 \alpha_{1}+\alpha_{2}} \oplus \mathfrak{p}_{3 \alpha_{1}+2 \alpha_{2}} \quad \text { and } \quad v_{o^{*}} F^{*}=\mathfrak{p}_{\alpha_{1}} \oplus \mathfrak{p}_{\alpha_{1}+\alpha_{2}} \oplus \mathfrak{p}_{2 \alpha_{1}+\alpha_{2}}=\boldsymbol{R}^{3}
$$

The action of $\mathfrak{s o}(3)$ on the normal space $\boldsymbol{R}^{3}$ is the standard one, which implies that the action of $S U(3)$ is of cohomogeneity one. Since there is only one $S U(3)$-action on $G_{2} / S O(4)$ up to conjugation, we conclude that the $S U(3)$-action on $G_{2} / S O(4)$ has indeed two totally geodesic singular orbits $C P^{2}$ and $S U(3) / S O(3)$. The bracket relations for root spaces imply that $v_{O^{*}} F^{*}$ is not a Lie triple system in $\mathfrak{p}$, which shows that $S U(3) / S O(3)$ is non-reflective. We again apply the construction described in Proposition 2.1 to get a second cohomogeneity one action on $M=G_{2}^{2} / S O(4)$. This time $S L(3, \boldsymbol{R})$ is the subgroup of $G_{2}^{2}$ which acts with cohomogeneity one on $G_{2}^{2} / S O(4)$, and the totally geodesic non-reflective singular orbit is $S L(3, \boldsymbol{R}) / S O(3)$.

(g) The orbit $F^{*}$ of the action of $G_{2} \times G_{2}$ through the identity $e$ of $\operatorname{Spin}(7)$ is clearly the totally geodesic subgroup $G_{2}=\left(G_{2} \times G_{2}\right) / \Delta G_{2}$, where $\Delta G_{2}$ is the diagonal embedding of $G_{2}$ in $G_{2} \times G_{2}$. On algebra level we have the following situation. Consider the Cartan decomposition of $\mathfrak{s p i n}(7) \oplus \mathfrak{s p i n}(7)=\mathfrak{k} \oplus \mathfrak{p}$ with

$$
\mathfrak{k}=\{(X, X) \mid X \in \mathfrak{s p i n}(7)\} \quad \text { and } \quad \mathfrak{p}=\{(X,-X) \mid X \in \mathfrak{s p i n}(7)\}
$$

and the decomposition $\mathfrak{s p i n}(7)=\mathfrak{g}_{2} \oplus \boldsymbol{R}^{7}$, where $\boldsymbol{R}^{7}$ is the 7-dimensional irreducible representation of $\mathfrak{g}_{2}$. Then we have

$$
T_{e} F^{*}=\left\{(X,-X) \mid X \in \mathfrak{g}_{2}\right\} \quad \text { and } \quad v_{e} F^{*}=\left\{(X,-X) \mid X \in \boldsymbol{R}^{7}\right\},
$$

and

$$
N_{\mathfrak{k}}\left(v_{e} F^{*}\right)=N_{\mathfrak{k}}\left(T_{e} F^{*}\right)=\left\{(X, X) \mid X \in \mathfrak{g}_{2}\right\}=\mathfrak{g}_{2} .
$$

Clearly, the slice representation of $N_{K}\left(T_{e} F^{*}\right)=G_{2}$ is transitive on the unit sphere in $v_{e} F^{*}=$ $\boldsymbol{R}^{7}$, which shows that the action of $G_{2} \times G_{2}$ on $\operatorname{Spin}(7)$ is indeed of cohomogeneity one. But $v_{e} F^{*}$ cannot be a Lie triple system, since otherwise $\left[v_{e} F^{*}, v_{e} F^{*}\right] \subset N_{\mathfrak{k}}\left(T_{e} F^{*}\right)=\mathfrak{g}_{2}$, which would imply that $\left(\mathfrak{s p i n}(7), \mathfrak{g}_{2}\right)$ is a symmetric pair, which is not true. Hence it follows that $F^{*}$ is non-reflective. We now construct the dual action according to Proposition 2.1, which is the action of $G_{2}^{C}$ on $S O(7, C) / S O(7)$. This action is of cohomogeneity one with a totally geodesic non-reflective singular orbit $G_{2}^{C} / G_{2}$.

We also have to consider the second singular orbit of the action of $G_{2} \times G_{2}$ on $\operatorname{Spin}(7)$. Let $z \in \operatorname{Spin}(7)$ be the generator of the center $\boldsymbol{Z}_{2}$ of $\operatorname{Spin}(7)$. Since $G_{2}$ has trivial center, $z$ is not contained in $F^{*}=G_{2} \subset \operatorname{Spin}(7)$, and hence the $\left(G_{2} \times G_{2}\right)$-orbit through $z$ is different 
from the orbit through $e$. Moreover, we have $\left(h_{1}, h_{2}\right) \cdot z=h_{1} z h_{2}^{-1}=z\left(h_{1} h_{2}^{-1}\right)$ for all $h_{1}, h_{2} \in G_{2}$, which shows that the $\left(G_{2} \times G_{2}\right)$-orbit through $z$ is the left translate in $\operatorname{Spin}(7)$ of the totally geodesic $\left(G_{2} \times G_{2}\right)$-orbit through $e$. Since left translation is an isometry, we see that the second singular orbit of the action is another totally geodesic non-reflective $G_{2}$ in $\operatorname{Spin}(7)$ (which is obviously not a subgroup). Since both singular orbits are conjugate via some left translation in $\operatorname{Spin}(7)$, the cohomogeneity one action on $S O(7, \mathrm{C}) / S O(7)$ constructed from the second singular orbit is orbit equivalent to the one constructed from the first one.

(h) The story for the action of $S U(3) \times S U(3)$ on $G_{2}$ is more or less the same as in the previous case. We have to replace $\mathfrak{s p i n}(7)$ by $\mathfrak{g}_{2}, \mathfrak{g}_{2}$ by $\mathfrak{s u}(3)$, and the decomposition $\mathfrak{s p i n}(7)=\mathfrak{g}_{2} \oplus \boldsymbol{R}^{7}$ by $\mathfrak{g}_{2}=\mathfrak{s u}(3) \oplus \boldsymbol{R}^{6}$, respectively. The dual action is the action of $S L(3, \boldsymbol{C})$ on $G_{2}^{C} / G_{2}$ with totally geodesic non-reflective singular orbit $S L(3, C) / S U(3)$. The second singular orbit goes through an element $z$ in the centralizer of $S U(3)$ in $G_{2}$, which is not contained in the center of $S U(3)$.

Summing up, we have now obtained the following theorem, which implies the main result in the introduction.

THEOREM 4.2. Let $M$ be a connected, irreducible Riemannian symmetric space of noncompact type and $F$ a totally geodesic non-reflective submanifold of $M$. Then $F$ is the singular orbit of a cohomogeneity one action on $M$ if and only if $F$ is one of the following totally geodesic non-reflective submanifolds:

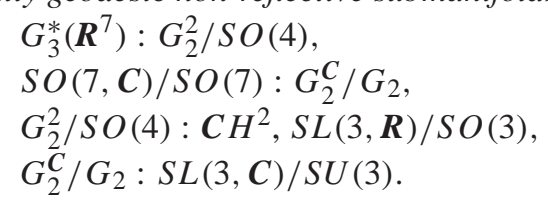

\section{REFERENCES}

[1] J. Berndt And M. BRÜCK, Cohomogeneity one actions on hyperbolic spaces, J. Reine Angew. Math. 541 (2001), 209-235.

[2] J. BERNDT AND H. TAMARU, Homogeneous codimension one foliations on noncompact symmetric spaces, J. Differential Geom. 63 (2003), 1-40.

[ 3 ] E. CARTAn, Familles de surfaces isoparamétriques dans les espaces à courbure constante, Ann. Mat. Pura Appl. IV. s. 17 (1938), 177-191.

[4] B. Y. Chen And T. Nagano, Totally geodesic submanifolds of symmetric spaces, I, Duke Math. J. 44 (1977), 745-755.

[ 5 ] R. Hermann, Totally geodesic orbits of groups of isometries, Nederl. Akad. Wetensch. Proc. Ser. A 65 (1962), 291-298.

[ 6 ] A. Kollross, A classification of hyperpolar and cohomogeneity one actions, Trans. Amer. Math. Soc. 354 (2002), 571-612.

[ 7 ] D. S. P. Leung, Errata: "On the classification of reflective submanifolds of Riemannian symmetric spaces" (Indiana Univ. Math. J. 24 (1974), 327-339), Indiana Univ. Math. J. 24 (1975), 1199.

[ 8 ] D. S. P. LEUNG, Reflective submanifolds. III. Congruency of isometric reflective submanifolds and corrigenda to the classification of reflective submanifolds, J. Differential Geom. 14 (1979), 167-177. 
UNIVERSITY COLLEGE

DEPARTMENT OF MATHEMATICS

CORK

IRELAND

E-mail address: berndt@ucc.ie
HIROSHIMA UNIVERSITY

DEPARTMENT OF MATHEMATICS

1-3-1 KAGAMIYAMA

HIGASHI-HIROSHIMA 739-8526

JAPAN

E-mail address: tamaru@math.sci.hiroshima-u.ac.jp 